\title{
Avaliação da qualidade do pré-natal em um hospital amigo da criança no interior do
}

\section{nordeste brasileiro}

\author{
Evaluation of prenatal care quality in a baby-friendly hospital in the brazilian northeast interior \\ Evaluación de la calidad de la atención prenatal en un hospital amigo da criança en el noreste de \\ Brasil
}

Recebido: 31/12/2021 | Revisado: 05/01/2022 | Aceito: 12/01/2022| Publicado: 14/01/2022

\author{
Cândida Regina Fonseca Chagas Rocha \\ ORCID: https://orcid.org/0000-0002-7394-3187 \\ Hospital e Maternidade Santa Isabel, Brasil \\ E-mail: candidaregina2007@gmail.com \\ Izailza Matos Dantas Lopes \\ ORCID: https://orcid.org/0000-0001-9752-5628 \\ Hospital e Maternidade Santa Isabel, Brasil \\ E-mail: izailzamatos@gmail.com
}

\begin{abstract}
Resumo
A atenção pré-natal apresenta um conjunto de medidas que visam levar a partos de recém-nascidos saudáveis, sem impactos negativos na saúde das mulheres. Inclui-se a promoção e a prevenção da saúde como também o diagnóstico e tratamento adequado dos problemas que possam surgir. O presente estudo tem como objetivo analisar o perfil epidemiológico das gestantes que realizaram o parto em um Hospital Amigo da criança no interior do estado de Sergipe, nos anos de 2018 e 2019, contribuindo para determinar a qualidade do pré-natal da população envolvida. Este estudo refere-se a um caso epidemiológico, retrospectivo, transversal e de caráter analítico, realizado através do levantamento de dados do banco do SINASC (Sistema de Informações de Nascidos Vivos) que se encontra na plataforma do DATASUS. Os resultados mostraram uma cobertura elevada da assistência pré-natal com mais de $90 \%$ das gestantes de parto termo iniciaram o acompanhamento do pré-natal antes da $16^{\mathrm{a}}$ semana gestacional $(98,1 \% ; \mathrm{n}=$ 2.972). Sobre o número de consultas de pré-natal consideradas adequadas pelo Ministério da Saúde, as gestantes que tiveram o parto termo realizaram $97,3 \%(\mathrm{n}=2.947)$ no mínimo seis consultas. Com relação à idade, as gestantes acima de 20 anos corresponderam a mais de $75 \%$ com filhos com o peso adequado ao nascimento (entre 2500 a 3999 g). A Classificação de Robson apresentada com maior frequência foi multípara, sem cesárea prévia, gestação única, bebê cefálico, trabalho de parto espontâneo. Concluiu-se que houve alta cobertura do pré-natal neste Hospital Amigo da Criança, porém apresenta adequação ao PHPN.
\end{abstract}

Palavras-chave: Gestantes; Atenção pré-natal; Saúde materno-infantil; Qualidade da assistência à saúde.

\begin{abstract}
Prenatal care presents a set of measures aimed at ensuring that healthy newborns are delivered without causing harm to women's health. Including health promotion and prevention, as well as proper diagnosis and treatment of difficulties that may arise. The current study aims to analyze the epidemiological profile of pregnant women who gave birth at a Baby-Friendly Hospital in the interior of Sergipe state, in the years 2018 and 2019, and to determine the quality of prenatal care for the population involved. This research is an epidemiological, retrospective, and crosssectional analytical study based on data collected from the Sinasc database on the Datasus platform. The findings revealed a high coverage of prenatal care with more than $90 \%$ of pregnant women at term birth starting prenatal care before the 16th gestational week $(98.1 \% ; n=2,972)$. According to the Ministry of Health's recommended number of consultations, 97.3 percent of pregnant women who delivered at full term $(\mathrm{n}=2947)$ received at least six consultations. Regarding age, pregnant women over 20 years old accounted for more than $75 \%$ of children with adequate birth weight (between 2500 and $3999 \mathrm{~g}$ ). Multipara, no previous cesarean, single pregnancy, cephalic baby, spontaneous labor were the most frequent Robson classifications. As has been noted, the Itabaiana municipality's Baby-Friendly Hospital has a high rate of prenatal care coverage; nonetheless, there are issues with the hospital's adequacy for the Prenatal and Birth Humanization Program.
\end{abstract}

Keywords: Pregnant women; Prenatal care; Maternal and child health; Quality of healthcare.

\section{Resumen}

Los cuidados prenatales presentan un conjunto de medidas destinadas a llevar a los partos de recién nacidos sanos, sin impactos negativos en la salud de las mujeres. Incluye la promoción y prevención de la salud, así como el diagnóstico y el tratamiento de los problemas que puedan surgir.El presente estudio tiene como objetivo analizar el perfil 
epidemiológico de las embarazadas que dieron a luz en un Hospital Amigo da Criança (HAC) en el interior del estado de Sergipe, en los años 2018 y 2019, contribuyendo a determinar la calidad del prenatal de la población involucrada.Este estudio se refiere a un caso epidemiológico, retrospectivo y transversal de carácter analítico realizado mediante el estudio de los datos de la base datos del Sinasc, que se encuentra en la plataforma de Datasus.Los resultados mostraron una alta cobertura de la atención prenatal con más del $90 \%$ de las gestantes a término iniciaron la atención prenatal antes de la $16^{\mathrm{a}}$ semana de gestación $(98,1 \%, \mathrm{n}=2.972)$.En cuanto al número de consultas prenatales consideradas adecuadas por el Ministerio de Salud, las embarazadas que tuvieron un parto a término, realizaron el 97,3\% ( $\mathrm{n}=2.947)$ al menos seis consultas.En cuanto a la edad, las mayores de 20 años correspondieran a más del 75\% con hijos con peso adecuado al nacer (entre 2500 y 3999g).La clasificación de Robson presentó con mayor frecuencia fue multípara, sin cesárea previa, gestación única, bebé cefálico, trabajo de parto espontáneo.Se concluye que existe una alta cobertura prenatal en este HAC, por lo que presenta adecuación al PHPN.

Palabras clave: Mujeres embarazadas; Atención prenatal; Salud materno-infantil; Calidad de la atención sanitária.

\section{Introdução}

A atenção pré-natal engloba um conjunto de medidas que visa levar a partos de recém-nascidos saudáveis, sem impactos negativos na saúde das mulheres. As consequências negativas são exemplificadas por meio de aspectos psicossociais e por meio de atividades educativas e preventivas cabíveis neste processo (Brasil, 2012). Diante desses aspectos, incluem-se à promoção e à prevenção da saúde como também o diagnóstico e o tratamento adequado dos problemas que possam surgir no processo de gestação. Assim, este tratamento é eficaz na redução da morbimortalidade relacionada ao ciclo gravídico-puerperal para as mães e seus recém-nascidos (Brasil, 2005; Carroli et al., 2001).

Inúmeros estudos demonstram a associação da atenção pré-natal com a prevenção de riscos na gestação, redução de complicações no parto, puerpério e complicações perinatais (Domingues et al., 2012; Vintzileos et al., 2002). Além desses fatores, muitas pesquisam apontam melhores condições de saúde do concepto, como melhor crescimento intrauterino, menor incidência de baixo peso ao nascer, redução da mortalidade materno-infantil e da morbimortalidade neonatal e perinatal (Brasil, 2002; Domingues et al., 2012; Gonzaga et al., 2016; Leal et al., 2017; Partridge et al., 2017; Segregur \& Segregur, 2017).

É importante frisar que o momento da primeira visita pré-natal bem como a frequência das visitas antenatais são usualmente referidos como possíveis elementos contribuintes na diminuição das taxas de morbimortalidade perinatal. O aumento no número de consultas de pré-natal é inversamente proporcional a taxas de mecônio no líquido amniótico, de índices de Apgar menores que (Vintzileos et al., 2002) no primeiro minuto e quinto minuto, de natimortalidade. Também, há estudo que identifica que existe incidência dos nascimentos pré-termo e de fetos de baixo peso (Morais et al., 1988).

No intuito de aprimorar a cobertura do pré-natal, o Ministério da Saúde por meio do Programa de Humanização no Pré-natal e Nascimento (PHPN) estabelece um pacote mínimo de procedimentos e exames a serem oferecidos a todas as gestantes durante a atenção pré-natal: (a) início da assistência até o quarto mês de gestação (16 a semana); (b) mínimo de seis consultas, preferencialmente uma no primeiro trimestre gestacional, duas no segundo e três no terceiro; (c) rotina de exames laboratoriais e vacinação, (d) atividades educativas e (e) consulta puerperal (Brasil, 2000). É importante lembrar de que a assistência pré-natal, muitas vezes, representa o primeiro contato das mulheres com os serviços de saúde e, por isso, deve ser organizada de forma a atender suas reais necessidades, por meio da utilização de conhecimentos técnico-científicos e dos recursos preconizados, em um contexto de humanização (Brasil, 2005 e OMS, 1987; WHO, 2016).

O estudo da qualidade da assistência pré-natal é um assunto bem relevante atualmente, visto que a maioria dos óbitos maternos e grande parte dos óbitos infantis ocorrem por causas evitáveis. Logo, são características sensíveis às ações desenvolvidas no pré-natal e que estão concentradas em países em desenvolvimento, como o Brasil. A avaliação do banco de dados do SINASC (Sistema de Informações de Nascidos Vivos) constitui-se atualmente em uma ferramenta essencial para o conhecimento do perfil epidemiológico, o planejamento e a avaliação das ações de saúde materno-infantil (Pessoa et al., 2009).

Por exemplo, o aumento das taxas de cesariana no Brasil se transformou em um grave problema de saúde pública 
impactando diretamente na saúde materno infantil. A OMS (Organização Mundial de Saúde) adotou a classificação de Robson com a finalidade de comparar variáveis do SINASC, relacionando as taxas de partos cesarianos com percentual de cesarianas anteriores. Tal comparação provocou a necessidade de haver indução no parto, gemelaridade, idade gestacional, apresentação do parto, entre outros (Lins et al., 2021).

Este estudo apresenta como objetivo principal analisar o perfil epidemiológico das gestantes que realizaram o parto no Hospital Amigo da Criança, que se localiza no interior do estado de Sergipe. Como objetivo específico, a fim de buscar respostas, este artigo pretende determinar a qualidade do pré-natal da população envolvida e classificar os partos no grupo de Robson.

\section{Metodologia}

Trata-se de um estudo epidemiológico, retrospectivo e transversal de caráter analítico. Na análise inferencial do presente estudo para verificar a relação entre as variáveis qualitativas foi utilizado o teste Qui-quadrado ( $\chi^{2}$ ), quando a frequência observada foi menor que 5 foi aplicado o teste Exato de Fisher. Em todos os testes de hipótese realizados, a conclusão foi obtida através da interpretação do p-valor. Adotando um nível de significância de 5\%, sempre que o p-valor calculado for menor que 0,05 diremos que há associação entre as variáveis analisadas. O software utilizado foi o R, versão 3.6.3.

As variáveis obtidas na pesquisa foram extraídas por meio do levantamento de dados do banco do SINASC, que se encontra na plataforma do Datasus. Como corpus, obteve-se dados de 8.171 gestantes no período de análise que compreende de janeiro de 2018 a dezembro de 2019.

O estudo usou como critério de inclusão as gestantes que realizaram partos na maternidade de Itabaiana, região localizada no interior de Sergipe. Exclui-se do estudo gestantes que realizaram partos em maternidades localizadas em outros interiores do estado de Sergipe e em outras regiões do Brasil. Para isto, realizou-se uma filtragem pelo próprio banco de dados.

As variáveis foram classificadas segundo o grupo de Robson: se a mulher já tem filhos; se já teve cesárea; se o bebê está de cabeça para baixo, sentado ou transverso; se é gestação única ou gemelar; se a idade gestacional está abaixo ou acima de 37 semanas; se o parto tem início espontâneo, é induzido ou se é feita uma cesárea eletiva.

O presente trabalho não foi submetido ao Comitê de Ética em Pesquisa (CEP), pois, segundo o inciso 3 do parágrafo único do Artigo $1^{\circ}$ da resolução número 510, de 07 de Abril de 2016, os dados foram extraídos de plataforma de domínio público, que não identificam os participantes da pesquisa.

\section{Resultados e Discussão}

No ano de 2019, o número de nascimentos em Itabaiana passou de 3.960 para 4.211 (entre 2018 e 2019), representando um acréscimo de 6,3\%. Em relação à escolaridade materna, a maioria das mães tem entre 8 a 11 anos de estudos, sendo que em 2019 foi de 53,2\% em 2019 e em 2018 53,4\%. A respeito do estado civil materno dos nascimentos ocorridos no município de Itabaiana, 71,3\% das mães tinham união consensual no ano de 2019 e em de 2018 houve o predomínio da união consensual em 70,2\%. (Tabela 1 ).

Pontua-se sobre o tipo de gravidez se foi única ou múltipla pelo fato de que no ano de 2018 o número de gravidez única foi de $99,7 \%$ e no ano de 2019 correspondeu a 99,5\%. Sobre a idade das gestantes, em 2018 a faixa etária era dos 20 a 34 anos e apresentou prevalência de 67,7\%. Em 2019, esse número correspondeu a 69,3\%. A porcentagem de gestantes crianças/adolescentes (entre 10 e 19 anos) foi de 21,5\%, em 2018 e no ano de 2019 foi $20,2 \%$. 
Ao relacionar estado civil e a idade da gestante, observa-se que 69,7\% (n=2916) das gestantes acima dos 20 anos eram casadas/união estável. Esse número foi de p<0,05. Ao analisar idade materna e tipo de parto, 79,7\% (n=3.359) das gestantes acima de 20 anos apresentaram partos normais com $\mathrm{p}<0,005$. (Tabela 3).

Tabela 1 - Características sociodemográficas das puérperas na maternidade do estudo nos anos de 2018 e 2019.

\begin{tabular}{|c|c|c|c|c|}
\hline \multirow{2}{*}{ Variável/Categoria } & \multicolumn{4}{|c|}{ Anos } \\
\hline & 2018 & 2018 & 2019 & 2019 \\
\hline Idade da mãe & Frequência & Porcentagem & Frequência & Porcentagem \\
\hline 10 a 19 anos & 850 & 21,5 & 851 & 20,2 \\
\hline 20 a 29 anos & 2028 & 51,2 & 2.198 & 52,2 \\
\hline 30 a 34 anos & 652 & 16,5 & 718 & 17,1 \\
\hline 35 a 49 anos & 430 & 10,9 & 443 & 10,5 \\
\hline Instrução da mãe & Frequência & Porcentagem & Frequência & Porcentagem \\
\hline Nenhuma & 16 & 0,4 & 18 & 0,4 \\
\hline 1 a 3 anos & 208 & 5,3 & 221 & 5,2 \\
\hline 4 a 7 anos & 1.336 & 33,7 & 1.434 & 34,1 \\
\hline 8 a 11 anos & 2.114 & 53,4 & 2.242 & 53,2 \\
\hline 12 anos e mais & 268 & 6,8 & 284 & 6,7 \\
\hline Ignorado & 18 & 0,5 & 12 & 0,3 \\
\hline Estado Civil & Frequência & Porcentagem & Frequência & Porcentagem \\
\hline Solteira & 533 & 13,5 & 536 & 12,7 \\
\hline Casada & 618 & 15,6 & 627 & 14.9 \\
\hline União Consensual & 2.778 & 70,2 & 3.001 & 71,3 \\
\hline Outras & 19 & 0,5 & 22 & 0,5 \\
\hline Ignorado & 12 & 0,3 & 25 & 0,6 \\
\hline Tipo de gravidez & Frequência & Porcentagem & Frequência & Porcentagem \\
\hline Única & 3.948 & 99,7 & 4.192 & 99,5 \\
\hline Dupla & 12 & 0,3 & 19 & 0,5 \\
\hline
\end{tabular}

Fonte: Autores.

Em relação à adequação do pré-natal, a maioria das gestações tiveram a quantidade de consulta pré-natal classificadas como mais que adequada, com percentual de 63,8\% em 2019 e 62,8\% em 2018 com um pequeno acréscimo de 1,0\%. A maioria das mulheres realizou de 7 ou mais consultas de pré-natal, sendo 68,5\% em 2019 e em 2018 67,8\%.

A respeito ao tipo de parto, o número de partos vaginais em 2019 foi de 63,2\%, consideravelmente maior que os partos cesáreos que representaram 36,8\%. No ano de 2018, o percentual de $65 \%$ refere-se ao número de partos vaginais, enquanto o número de partos cesáreos em 2018 foram 35,0\% (Tabela 2). Em relação às características dos recém-nascidos, observou-se o maior número de crianças com peso entre 2.500 a 3.999 g, sendo 90,1\% em 2019 e o mesmo valor percentual em 2018 (Tabela 2). 
Tabela 2 - Características do pré-natal e parto das puérperas na maternidade do estudo nos anos de 2018 e 2019.

\begin{tabular}{ccccc}
\hline Adeq quant pré-natal & Frequência & Porcentagem & Frequência & Porcentagem \\
Não fez pré-natal & 4 & 0,1 & 4 & 0,1 \\
Inadequado & 716 & 18,1 & 710 & 16,9 \\
Intermediário & 360 & 9,1 & 425 & 10,1 \\
Adequado & 391 & 9,9 & 366 & 8,7 \\
Mais que adequado & 2.464 & 62,2 & 2.685 & 0,5 \\
Não Classificados & 25 & 0,6 & 21 & Porcentagem \\
Tipo de parto & Frequência & Porcentagem & Frequência & 63,2 \\
Vaginal & 2.574 & 65,0 & 2.660 & 36,8 \\
Cesáreo & 1.386 & 35,0 & 1.551 & Porcentagem \\
Consult pré-natal & Frequência & Porcentagem & Frequência & 0,5 \\
Nenhuma & 27 & 0,7 & 22 & 25,1 \\
De 1 a 3 consultas & 188 & 4,7 & 215 & 68,5 \\
De 4 a 6 consultas & 1.061 & 26,8 & 1.089 & 2.885 \\
7 ou mais consultas & 2.684 & 67,8 & Prequência & 3,7 \\
Peso ao nascer & Frequência & Porcentagem & 157 & 90,1 \\
Até $2499 \mathrm{~g}$ & 121 & 3,1 & 3.794 & 6,2 \\
2500 g a 3999 g & 3.568 & 90,1 & 260 & \\
4000 g e mais & 271 & 6,8 & & \\
\hline
\end{tabular}

Fonte: Autores.

Mais de $90 \%$ das gestantes de parto termo iniciaram o acompanhamento do pré-natal antes da $16^{\text {a }}$ semana gestacional $(98,1 \% ; \mathrm{n}=2.972)$, principalmente as gestantes que possuíam união consensual/casadas ( $\mathrm{p}<0,05)$, caracterizando assim pré natal adequado (como é preconizado pelo programa PHPN). Em relação ao número de consultas de pré-natal, as gestantes que tiveram o parto termo $97,3 \%(n=2.947)$ realizaram as seis consultas mínimas preconizadas pelo Ministério da Saúde.

Ao analisar a faixa etária, observa-se que as gestantes acima de 20 anos que apresentaram parto termo e pós termo $(81 \%, n=2.188)$ realizaram mais de 7 consultas de pré-natal. Sobre a relação de adequação do pré-natal por faixa etária, as gestantes de parto termo e pós termo acima de 20 anos realizaram um pré-natal adequado e mais que adequado com o percentual de 58,84\% $(\mathrm{n}=2.476, \mathrm{p}<0,005)$. Esse resultado sugere que um pouco mais da metade das gestantes conseguiram realizar mais de 6 consultas de pré-natal e iniciar as consultas antes da $16^{\mathrm{a}}$ semana gestacional.

Identificou-se sobre o peso de nascimento do recém-nascido em relação ao tempo de gestação do parto: mais de $90 \%$ das gestantes de parto termo e pós termo tiveram filhos com peso adequado para o nascimento entre 2500 a 3999 g com $\mathrm{p}<0,05$. Ao comparar o peso de nascimento do recém-nascido e a idade da gestante, inferiu-se que gestantes acima de 20 anos corresponderam a mais de $75 \%$ com filhos com peso adequado ao nascimento (entre 2500 a 3999, p<0,05).

Ao relacionar duração da gestação e o Apgar ao nascimento do recém-nascido, observa-se que o Apgar no primeiro minuto entre 8 e 10 foi prevalente em aproximadamente 91,6\% $(n=3.776)$ das gestantes de parto termo e pós termo. A respeito da relação entre a duração da gestação e o Apgar no quinto minuto, o índice entre 8 e 10 corresponde aproximadamente 96,8\% $(n=3972)$ das gestantes de parto termo e pós termo. Com isso, essa porcentagem apresenta um bom índice de Apgar, o que evidencia uma boa avaliação do estado geral e vitalidade do recém-nascido.

Entre a idade materna e o Apgar no primeiro minuto, observou que 74,9\%(n=3.098) dos filhos das mães acima de 20 anos apresentou o Apgar entre 8 e 10 no primeiro minuto. Sobre a relação entre o Apgar no quinto minuto e a idade materna, pode-se inferir que 79\% ( $\mathrm{n}=3.266)$ dos filhos de mães acima de 20 anos tiveram o Apgar no quinto minuto entre 8 e 10. 
Tabela 3 - Qualidade do pré-natal na maternidade do estudo comparados com duração da gestação, idade materna, nos anos de 2018 e 2019.

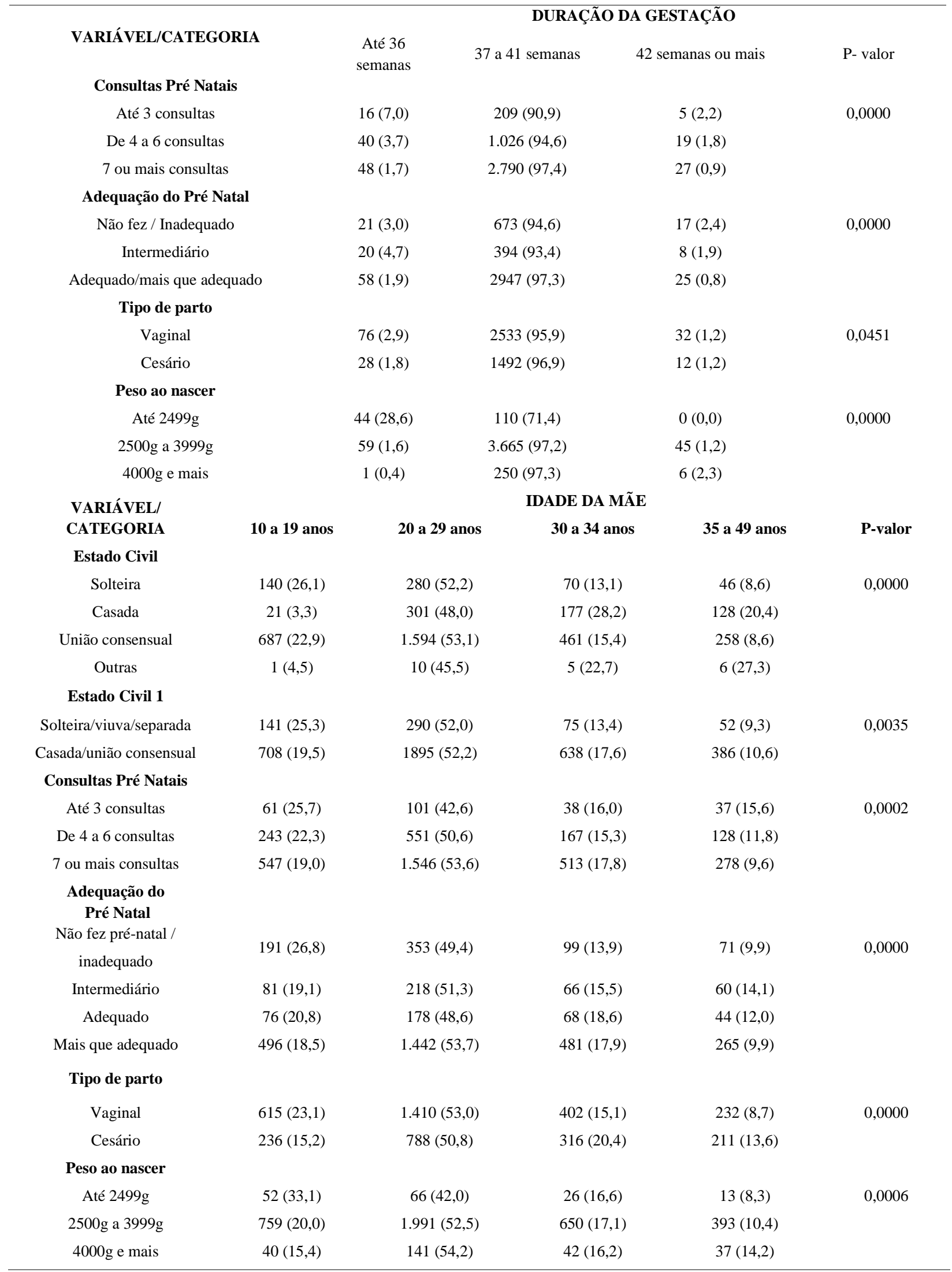

Fonte: Autores. 
Gráfico 1: Percentual de nascidos vivos segundo grupo Robson - Itabaiana - 2019.

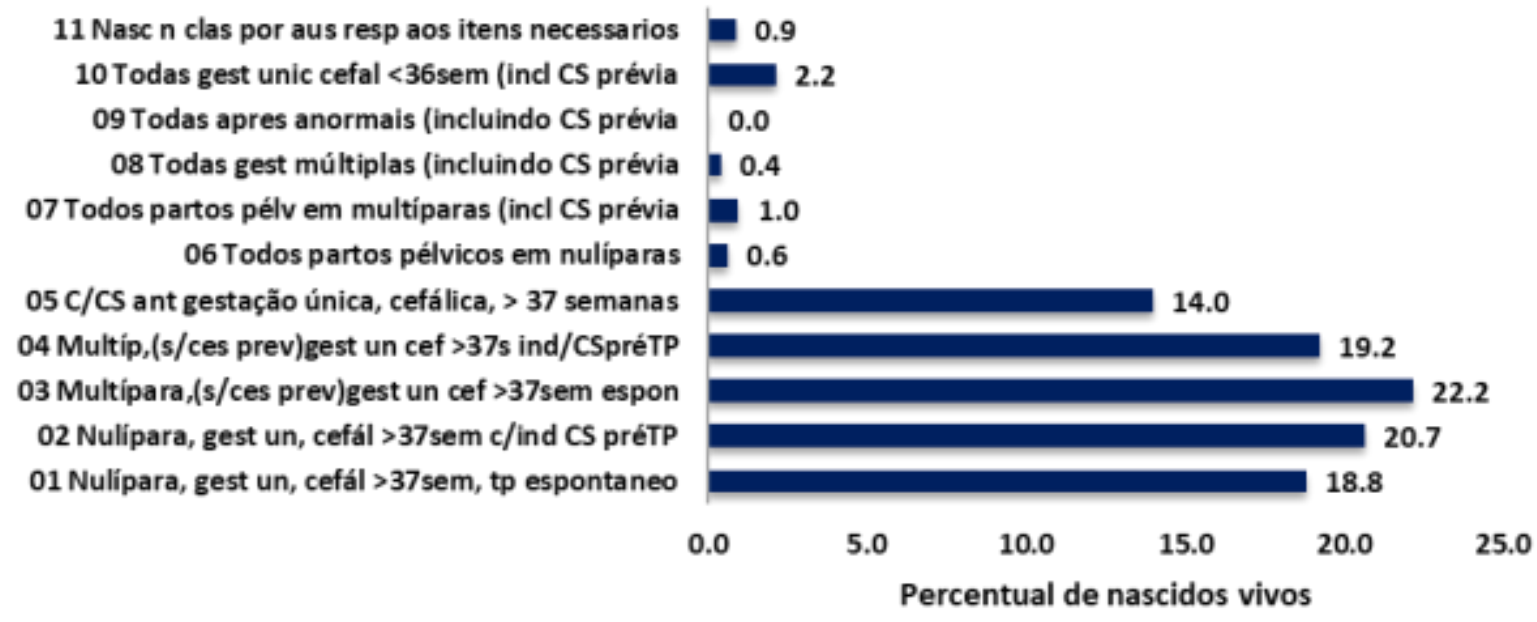

Fonte: DataSus.

A respeito da Classificação de Robson (Gráfico 1), observada neste estudo, esse resultado apresenta com maior frequência a classificação 3 (multípara, sem cesárea prévia, gestação única, bebê cefálico, trabalho de parto espontâneo) 22,2\%; seguida de classificação 2 (primeiro filho, gestação única, bebê cefálico e trabalho de parto induzido ou cesárea antes do parto) 20,7\%; depois a classificação 4 (multípara, sem cesárea anterior, gestação única, com mais 37 semanas, bebê cefálico, trabalho de parto induzido ou cesárea antes do trabalho de parto) 19,2\%; classificação 1 (primeiro filho, gestação única, com mais 37 semanas, bebê cefálico, trabalho de parto de início espontâneo) 18,8\%.

\section{Discussão}

A maioria das gestantes do Hospital Amigo da Criança tinha entre 20 e 34 anos de idade, possuía entre 8 a 11 anos de estudo, vivia com companheiro e apresentava gestação única. Observou-se que grande parte das gestantes apresentavam uma baixa escolaridade (Ensino Fundamental incompleto) na qual está associada aos altos percentuais de mortalidade materna e perinatal, uma vez que a escolaridade materna pode ser uma das peças fundamentais durante o período gravídico devido à influência no comportamento das gestantes.

A escolaridade da gestante é um fator importante na assistência pré-natal, uma vez que, de acordo com pesquisas, (Rodrigues et al., 2012; Santos et al., 2015) das gestantes com baixa escolaridade somado a um baixo nível socioeconômico costumam apresentar maior índice de absenteísmo - além de apresentar hábitos inadequados durante o período gestacional.

Sobre o status marital, a presença de um parceiro como ocorreu na grande parte dos casos é fundamental durante todo o processo gravídico, principalmente quando o companheiro acompanha sua mulher durante as consultas pré-natais, o que permite um maior preparo do casal para o parto, por levar em conta que a gestação é uma fase repleta de transformações (Brasil, 2012; Santos et al., 2009). Além disso, as mulheres solteiras e sem apoio social apresentam um maior risco para se adoecer, ter estresse psicológico elevado, ansiedade em relação à maternidade (motivada tanto pela ausência de um companheiro como pela desestruturação familiar) e até mesmo devido às alterações gravídicas. Todos esses fatores incidem e aumentam a chance de apresentarem partos prematuros (Brasil, 2012).

O número de consultas de pré-natal realizadas pelas mães que tiveram seus filhos no Hospital Amigo da Criança no município de Itabaiana apresentou valores satisfatórios, independentemente das características sociais e reprodutivas das mulheres. A adequação dessa assistência é considerada satisfatória, segundo o primeiro critério para a avaliação das 
recomendações do PHPN4 (IG na primeira consulta e número de consultas de acordo com a idade gestacional), pois, ao analisar os dados do SINASC, 71,3\% das gestantes iniciaram o pré-natal antes da $16^{\mathrm{a}}$ semana gestacional, o que indica busca da assistência do pré-natal precocemente como preconizado pela OMS no primeiro trimestre da gravidez

Na primeira consulta pré-natal, a idade gestacional revela a captação precoce das gestantes pelos serviços de saúde. Ao iniciar o acompanhamento pré-natal tardiamente, essa relação fica relevada o quanto há fragilidade na organização dos serviços de saúde e lacunas no processo de trabalho quanto à captação de gestantes de forma precoce e quanto à conscientização da população (Anvesa etl al., 2012). Iniciar precocemente o pré-natal permite antecipar a detecção de riscos e prevenir complicações. A relação de cada intervenção durante a gestação com o declínio da mortalidade materna no mundo ainda não está bem estabelecida, embora sejam eficazes para a melhoria da saúde em geral (Olivier, 1999).

Entretanto, 27,2\% das gestantes não tiveram o número mínimo de consultas previstas para a idade gestacional - o que impactou negativamente na saúde materna infantil. Estas consultas correspondem a uma no primeiro trimestre, duas no segundo e três no terceiro trimestre. Em um estudo realizado no Rio de Janeiro-RJ (Domingues et al., 2012), foi observado que $74,4 \%$ das gestantes iniciaram o pré-natal antes da $16^{\mathrm{a}}$ semana de gestação e 79,2\% realizaram número adequado de consultas. Este resultado foi um pouco inferior ao encontrado em Maringá (81,2\% e 85,4\%, respectivamente). De qualquer forma, os dados corroboram com o presente estudo, pois a diferença entre as porcentagens foi quase inexistente (Melo, Oliveira \& Mathias, 2015).

Definir o número ideal de consultas no pré-natal é importante para a elaboração de protocolos assistenciais e planejamento dos recursos em saúde. Na teoria, um número maior de consultas pode significar que a gestante terá mais oportunidades de receber cuidados preventivos e de promoção de saúde, especialmente em gestações de maior risco e com maior probabilidade de afetar os desfechos perinatais (Wehby et al., 2006). No entanto, a discussão mais relevante é sobre a qualidade dessas consultas e a utilização desses contatos com os serviços para a realização de cuidados efetivos em saúde. Apenas a quantidade de consultas isoladamente não reproduz a realidade da qualidade de uma assistência pré-natal.

As crianças e adolescentes neste estudo obtiveram um índice significativo de um pré-natal realizado de forma inadequada/não adequada $(26,8 \%, \mathrm{n}=191)$ e intermediário $(19,1 \%, \mathrm{n}=81)$. Ou seja, esses resultados apontam que não se realizou um pré-natal da forma que é preconizada pelo Ministério da Saúde. As gestantes crianças/adolescentes (entre 10 a 19 anos de idade) apresentaram menor cobertura da assistência pré-natal. Tais resultados também foram evidenciados por outros estudos anteriores (Cesar et al., 2011; Pereira et al., 2006; Viellas et al., 2014, Wiemann et al., 1997;).

Tal conduta pode estar relacionada ao desconhecimento sobre os serviços de pré-natal disponíveis, falta de autonomia decisória e estigmas sociais, uma vez que a gestação na adolescência tem associação com estado civil não-casada, demonstrando, assim, a necessidade de estratégias diferenciadas para esse grupo etário (Miranda et al., 2013; Lessa et al., 2006).

No presente estudo, a qualidade do cuidado pré-natal está associada ao nascimento prematuro. No Hospital Amigo da Criança, observou-se que os partos prematuros apresentam um baixo índice e evidenciam que as gestantes de parto prematuro que realizaram o parto nesta unidade fizeram um pré-natal com quantidade insatisfatória de consultas; ou não fizeram prénatal; ou realizaram pré-natal de maneira inadequada, pois apresentaram um valor de 7,7\% do total dos partos realizados nesta unidade. Este resultado mais uma vez demonstra que a qualidade do cuidado pré-natal, especialmente no que tange à captação precoce da gestante e ao número adequado de consultas é fundamental para assegurar a realização de todos os procedimentos e condutas a tempo durante a gestação desempenhando papel importante na prevenção do nascimento prematuro (Brasil, 2006; Brasil, 2011; Sarruya et al., 2004).

O pré-natal adequado na prevenção do nascimento prematuro é de extrema relevância para avaliar a qualidade e detectar as lacunas do cuidado. O conhecimento das características maternas, associado ao cuidado inadequado, é primordial 
para intervir sobre elas (Victora et al., 2010). É relevante lembrar de que a importância do cuidado pré-natal e da prematuridade na mortalidade neonatal e infantil sinalizado no presente estudo possui taxas menores que em outras regiões do país.

Um estudo feito em um município da região sul do Brasil mostrou dados retrospectivos da mortalidade neonatal em período de 10 anos. Com isso, esse estudo apontou, dentre outros fatores, que a prematuridade e o número insuficiente de consultas no pré-natal são principais fatores determinantes para o óbito nesta fase da vida (Ferrari et al., 2013). Em um outro estudo que usou estimativas e dados mundiais da mortalidade neonatal e infantil, inferiu-se que o nascimento prematuro é a principal causa de mortalidade em menores de 5 anos de idade. Também, identificou-se a importância de outras causas que poderiam ser evitadas com um pré-natal adequado, como o tétano e a AIDS, reforçando a necessidade de uma assistência prénatal que contemple a equidade e o acesso integral à gestante (Segregur \& Segregur, 2017).

A Classificação de Robson tem um papel fundamental e ímpar na mudança do padrão obstétrico vigente no Brasil. Neste estudo, os resultados sugerem, com maior frequência, a classificação 3 em 22,2\% (multípara, sem cesárea prévia, gestação única, bebê cefálico, trabalho de parto espontâneo). O seu uso na rotina hospitalar permitirá uma análise objetiva e eficaz, especialmente nos grupos os quais não apresentam risco à realização de cesáreas por apresentar gestações com feto único, cefálico e a termo (Aguemi, 2021).

Com os resultados de uma pesquisa documental, a Classificação de Robson se apresenta como um instrumento que auxilia na identificação e análise das características obstétricas das parturientes atendidas em determinada unidade (Oliveira et al., 2020). Este estudo apresenta como objeto subsidiar uma investigação dos partos por grupos e comparar as taxas de cesárea dentro do estabelecimento e entre diferentes serviços de saúde. A partir disso, é possível desenvolver políticas institucionais e estratégias para melhoria da conduta clínica prestada a gestante e, consequentemente, o seu filho (Lins et al., 2021).

\section{Considerações Finais}

Este estudo permitiu inferir que o Hospital Amigo da Criança no município de Itabaiana atingiu uma boa cobertura da assistência pré-natal. Entretanto, há vários dados que indicam ser de qualidade e adequação insuficientes ao PHPN. Os principais problemas identificados foram a falta da avaliação de: (i) exames e vacinas realizados no pré-natal pelas gestantes; (ii) a falta de dados sobre as orientações, inclusive sobre a maternidade de referência para o parto, peregrinação no anteparto e falta de priorização das gestantes de maior risco; (iii) informações sobre as consultas puerperais.

Destaca-se que as limitações deste estudo estão relacionadas à confiabilidade de dados do banco do SINASC, que se encontra na plataforma do Datasus, pois é um sistema alimentado por meio das informações da Certidão de Nascido Vivo.

Cabe salientar que as informações valiosas que poderiam ser incluídas ou estarem interligadas ao SINASC estão disponíveis na plataforma do SISPRENATAL, inserido dentro da Rede Cegonha. Esta rede, infelizmente, não tem domínio público, logo não há como captar as informações contidas neste sistema. O SISPRENATAL permite cadastrar a gestante, monitorar e avaliar a atenção do pré-natal ao puerpério prestadas pelos serviços de saúde a cada gestante e recém-nascido, desde o primeiro atendimento na Unidade Básica de Saúde até o atendimento hospitalar de alto risco. Sugere-se que em pesquisas futuras os pesquisadores consigam unir as informações destes dois banco de dados SINASC e SISPRENATAL para que através das informações contidas nestes bancos os pesquisadores consigam definir melhorias no planejamento e execução do pré-natal e que impactem diretamente tanto na morbimortalidade materno-infantil como também na melhoria da qualidade da saúde materno-infantil no Brasil.

Ressalta-se que é imprescindível para a redução da morbimortalidade materno-infantil haver um acompanhamento do início do pré-natal até a sua finalização para que não haja partos de maneira precoce. A utilização de recursos preconizados pelo Ministério da Saúde, como ações de promoção, de prevenção da saúde, de diagnóstico e de tratamento adequado dos 
problemas, pode vir a ocorrer no período gravídico-puerperal de maneira rápida e efetiva, para que isso não prejudique nem a saúde da gestante e nem do seu filho.

Por fim, houve uma alta cobertura do pré-natal neste Hospital Amigo da Criança, localizado no município de Itabaiana. Porém, apresentou-se problemas relacionados a sua adequação ao PHPN e os dados do SINASC deveriam ser ampliados ou compilados com o sisprenatal para uma melhor avaliação da qualidade do pré-natal.

\section{Referências}

Aguemi, A. K. (2021). Indicadores maternos para monitorar hospitais da Rede Cegonha: uma proposta. Ciência \& Saúde Coletiva, $26(3), 781-787$.

Anversa, E. T. R, Bastos, G. A. N, Nunes, L. N, Pizzol, T. S. D. (2012). Qualidade do processo da assistência pré-natal:unidades básicas de saúde e unidades de Estratégia Saúde da Família em município no Sul do Brasil. Caderno de Saúde Pública, 28(4), 789-800.

Brasil. (2012). Ministério da Saúde. Atenção ao pré-natal de baixo risco. Brasília: Ministério da Saúde, Caderno de Atenção Básica no 32.

Brasil. (2000). Ministério da Saúde. Portaria no 569, de 1o de junho de 2000. Institui o Programa de Humanização no Pré-natal e Nascimento (PHPN). Diário Oficial da União.

Brasil. (2005). Ministério da Saúde. Pré-natal e Puerpério: atenção qualificada e humanizada - manual técnico. Brasília: Ministério da Saúde, Série A, Caderno $\mathrm{n}^{\circ} 5$.

Brasil. (2011). Ministério da Saúde. Portaria n. 1.459, de 24 de junho de 2011. Institui, no âmbito do Sistema Único de Saúde - SUS a Rede Cegonha. Brasília: Ministério da Saúde.

Brasil. (2006). Ministério da Saúde. Secretaria de Atenção à Saúde, Departamento de Ações Programáticas Estratégicas. Área Técnica de Saúde da Mulher. Pré-natal e puerpério: atenção qualificada e humanizada: manual técnico. Brasília: Ministério da Saúde.

Carroli, G, Rooney, C. \& Villar, J. (2001). How effective is antenatal care in preventing maternal mortality and serious morbidity? An overview of the evidence. Paediatr Perinat Epidemiol, 15(1), 1-42.

Cesar, J. A, Sassi, R. A. M, Chica, D. A. G, Mano, P. S, \& Goulart, S. M. F. (2011). Características sociodemográficas e de assistência à gestação e ao parto no extremo sul do Brasil. Caderno de Saúde Pública, 27(5), 985-994.

Domingues, R. M. S. M, Hartz, Z. M. A, Dias, M. A. B. \& Leal, M. C. (2012). Avaliação da adequação da assistência pré-natal na rede SUS do Município do Rio de Janeiro, Brasil. Caderno de Saúde Pública, 28(3):425-437.

Ferrari, R. A. P, Bertolozzi, M. R, Dalmas, J. C, \& Girotto, E. (2013). Determining factors for neonatal mortality in a city in the southern region of Brazil. Rev Escola de Enfermagem USP, 47(3):531-38.

Gonzaga, I. C. A, Santos, S. L. D, Silva, A. R. V, \& Campelo, V. (2016). Atenção pré-natal e fatores de risco associados à prematuridade e baixo peso ao nascer em capital do nordeste brasileiro. Ciência e Saúde Coletiva, 21(6):1965-1974.

Leal, M. C, Bittencourt, S. D. A, Torres, R. M. C, Niquini, R. P, \& Souza Jr., P. R. B. (2017). Determinantes do óbito infantil no Vale do Jequitinhonha e nas regiões Norte e Nordeste do Brasil. Revista de Saúde Pública, 51(12):1-9.

Lessa, F. S, Cunha, A. A, Pinhal, I. M. C, Bornia, R. G, \& Nejaim, J. E. (2006). A adolescência como fator de risco social na gravidez. Adolescência e Saúde, $3(2): 29-32$.

Lins, J. J. A, De Lima, M. E. T, Dos Santos, S. A. A, \& E Silva, J. M. (2021). A aplicação da Classificação de Robson nas maternidades brasileiras como ferramenta para redução das taxas de cesariana: uma revisão de literatura. Research, Society and Development, 10(13), 1-10.

Melo, E. C, Oliveira, R. R, \& Mathias, T. A. F. (2015). Fatores associados à qualidade do pré-natal:uma abordagem ao nascimento prematuro. Revista Escola de Enfermagem USP,49(4), 540-549.

Miranda, F. R. D, Taquette, S. R, Monteiro, D. L. M, Blanco, M. N, \& Rodrigues, A. O. (2013). Pré-natal na adolescência: uma revisão crítica. Adolescência e Saúde, 10(1), 43-50.

Morais, E. N, Alflen, T. L, Spara, P, \& Beitune, P. E. (1988). Momento e frequiência das visitas de pré-natal: repercussões sobre os nascimentos pré-termo. Revista Brasileira de ginecologia, 20(1), 25-32.

Oliveira, E. G, Ferreira, L. M, Sales, J. L, Marins, M. A. R, Knupp, V. M. A. O, \& Quitete, J. B. (2020). Perfil das mulheres submetidas à cesariana segundo Classificação De Robson: pesquisa de campo. Online Brazilian Journal of Nursing, 18(3).

Olivier, W. (1999). Is safe motherhood an orphan initiative? Lancet, 354(9182), 940-943.

OMS - Organização Mundial da Saúde. (1987). La Prevención de la Tragedia de las Muertes Maternas - Informe sobre la "Conferencia Internacional sobre la Mortalidad Materna". Nairobi: Banco Mundial/OMS/UNFPA, 1987.

Partridge, S, Balayla, J, Holcroft. C. A, \& Abehaim, H. A. (2012). Inadequate prenatal care utilization and risks of infant mortality and poor birth outcome: a retrospective analysis of 28,729,765 U.S. deliveries over 8 years. American Journal of Perinatology, 29(10):787-793. 
Research, Society and Development, v. 11, n. 1, e53111125277, 2022

(CC BY 4.0) | ISSN 2525-3409 | DOI: http://dx.doi.org/10.33448/rsd-v11i1.25277

Pereira, P. H. G, Antón, A. G. S, Vieira Jr, W. S, Domingues, R. A. D, Melo, A. L, Farias, C. S, Paula, T. F, Carvalho, R. P, Araújo, P. R, Godoy, R. B, \& Muza, G. M. (2005). Fatores associados ao acesso tardio ao pré-natal do Centro de Saúde nº 1 do Paranoá, 2005. Comunicação, Ciência e Saúde, 17(2):101110.

Pessoa, I. N, Menezes, E. D, Ferreira, T. F, Dotto, L. M. G, \& Bessa, L. F. (2009). Percepção de puérperas sobre assistência de enfermagem na gravidez. Ciência, Cuidado e Saúde, 8(2):236-241

Rodrigues, L. S, Batista, R. F. L, Sousa, A. C. V, Cantanhede, J. G, \% Costa, L. C. (2012). Caracterização dos recém-nascidos pré-termos nascidos em São Luís - MA no período de 2006 a 2010: análise do SINASC. Cadernos de Pesquisa, 19(3), 97-106.

Santos, G. H. N, Martins, M, G, Sousa, M. S, \& Batalha, S. J. C. (2009). Impacto da idade materna sobre os resultados perinatais e via de parto. Revista Brasileira de Ginecologia e Obstetrícia, 31(7), 326-334.

Santos, J. O, Pacheco, T. S, de Oliveira, P. S, Pinto, V. L, Gabrielloni, M. C, \& Barbieri, M. (2015). The obstetrical and newborn profile of postpartum women in maternities in São Paulo. Revista Pesquisa Cuidado é Fundamental, 7(1), 1936-1945.

Šegregur, J, \& Šegregur, D. (2017). Antenatal characteristics of Roma female population in Virovitica-Podravina County, Croatia. Slovenian Journal of Public Health, 56(1):47-54.

Serruya, S. J, Lago, T. G, \& Cecatti, J. G. (2004). Avaliação preliminar do Programa de Humanização no Pré-Natal e Nascimento no Brasil. Revista Brasileira de Ginecologia e Obstetrícia, 26(7), 517-25.

Victora, C. G, Matijasevich, A, Silveira, M. F, Santos, I. S, Barros, A. J. D, \& Barros, F. C. (2010). Socioeconomic and ethnic group inequities in antenatal care quality in the public and private sector in Brazil. Health Policy Planning, 25(4), 253-261.

Viellas, E. F, Augusto, M, Dias, B, Viana, J, \& Bastos, M. H. (2014). Assistência pré-natal no Brasil. Caderno de Saúde Pública, 30(1), 85-100.

Vintzileos, A. M, Ananth, C. V, Smulian, J. C, Scorza, W, \& Knuppel, R. A. (2002). The impact of prenatal care on neonatal deaths in the presence and absence of antenatal high-risk conditions. American Journal of Obstetrics and Gynecology, 186(5), 1011-1016.

Wehby, G. L, Murray, J. C, Castilla, E. E, Lopez-Camelo, J. S, \& Ohsfeldt, R. L. (2009). Prenatal care effectiveness and utilization in Brazil. Health Policy Planning, (24), 175-88.

Wiemann, C. M, Berenson, A. B, Pino, L. G, McCombs, S. L. (1997). Factors associated with adolescents' risk for late entry into prenatal care. Family Planning Perspectives, 29(6), 273-276.

WHO - World Health Organization. (2016). WHO recommendations on antenatal care for a positive pregnancy experience. Geneva: WHO. http://apps.who.int/iris/bitstre am/10665/250796/1/9789241549912-eng.pdf?ua=1. 